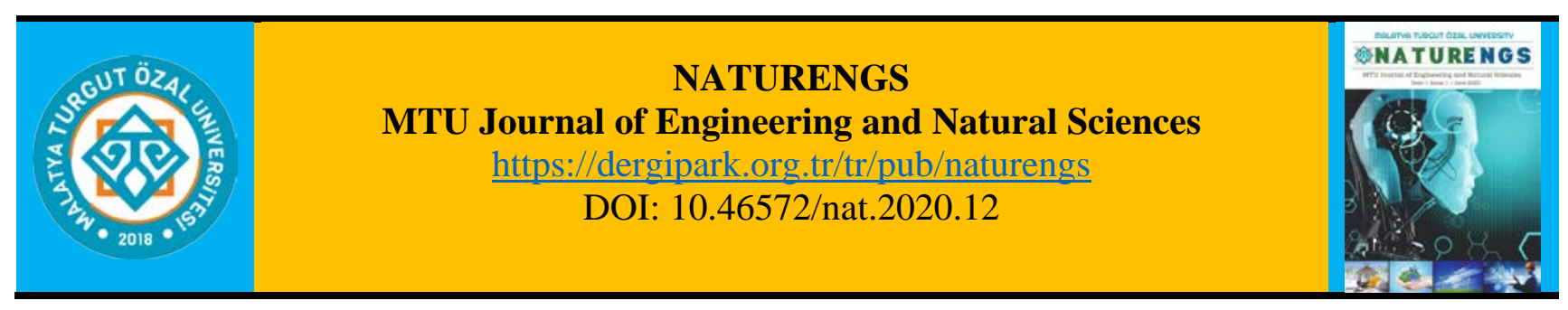

\title{
The Generalized Odd Nakagami-G Family of Distributions: Properties and Applications
}

\author{
Ibrahim ABDULLAHI ${ }^{1 *}$, Job OBALOWU² \\ ${ }^{1}$ Department of Mathematics and Statistics, Faculty of Science, Yobe State University, 620, Yobe, Nigeria. \\ ${ }^{2}$ Department of Statistics, Faculty of Physical Science, University of Ilorin, 240003, Kwara, Nigeria.
}

(Received: 15.08.2020; Accepted: 01.10.2020)

\begin{abstract}
In this study, the Generalized Odd Nakagami-G distribution has been empirically investigated using Frechet distribution as the baseline distribution. Some mathematical statistics properties viz: quantile function, moments, probability-weighted moment, entropies, and order statistics were derived among others. The method of maximum likelihood estimation is suitable for the derivation of estimators of the distribution parameters. And the Generalized odd Nakagami Frechet (GONak-Fr) distribution gives the best fit vis-a-vis its competitors via application to real life data sets.
\end{abstract}

Keywords: GONak-G, GONak-Fr, Probability-weighted moment, Entropies, Order statistics.

\section{INTRODUCTION}

There have been recent developments focus on generalized odd classes of continuous distributions by adding at least one shape parameters to the baseline distribution, has led mathematical statistician researcher to make of the new flexible distributions and studying the properties of these distributions and using these distributions to model data in many applied areas which include engineering, biological studies, environmental sciences and economics. Numerous methods for generating new families of distributions have been proposed [1] by many researchers.

The beta-generalized family of distribution was developed, Kumaraswamy generated a family of distributions [2], Beta-Nakagami distribution [3], Weibull generalized family of distributions [4], Additive Weibull generated distributions [5], Kummer beta generalized family of distributions [6], the Exponentiated-G family [7], the Gamma-G (type I) [8], the Gamma-G family (type II) [9], the McDonald-G [10], the Log-Gamma-G [11], A new beta generated Kumaraswamy Marshall-Olkin-G family of distributions with applications [12], Beta MarshallOlkin-G family [13] and Logistic-G family [14].

In this paper, the so-called generalized odd Nakagami generator and the corresponding family of distributions are called generalized odd Nakagami (GONak-G) family of distributions. To the best of the researcher's knowledge, it is new in the literature.

*Corresponding Author: ibworld@ysu.edu.ng

ORCID number of authors: ${ }^{1}$ 0000-0002-7280-3035, ${ }^{2}$ 0000-0001-5232-1509 


\section{CONSTRUCTIONS OF THE GENERALIZED ODD NAKAGAMI DISTRIBUTION}

The Generalized Odd Nakagami-G distribution (GONak-G) would be made

$$
\begin{aligned}
& F(x ; \lambda, \beta, \tau, \eta)=\int_{0}^{\frac{G(x ; \eta)^{\tau}}{1-G(x ; \eta)^{\tau}}} \frac{2 \lambda^{\lambda}}{\Gamma(\lambda) \beta^{\lambda}} t^{2 \lambda-1} e^{-\frac{\lambda t^{2}}{\beta}} d t \\
& F(x . \lambda, \beta, \eta)=\gamma_{*}\left\{\lambda, \frac{\lambda}{\beta}\left[\frac{G(x ; \eta)^{\tau}}{\left(1-G(x ; \eta)^{\tau}\right)}\right]^{2}\right\}
\end{aligned}
$$

where

$$
\gamma_{*}\left\{\lambda, \frac{\lambda}{\beta}\left[\frac{G(x ; \eta)^{\tau}}{\left(1-G(x ; \eta)^{\tau}\right)}\right]^{2}\right\}=\frac{1}{\Gamma(\lambda)}\left\{\lambda, \frac{\lambda}{\beta}\left[\frac{G(x ; \eta)^{\tau}}{\left(1-G(x ; \eta)^{\tau}\right)}\right]^{2}\right\}
$$

where $\lambda, \beta, \tau>0$ are three additional parameters, $\eta$ is the parameter for baseline $G(x)$ and $\gamma(\sigma, x)=\int v^{\sigma-1} e^{-v} \partial v$ is the incomplete gamma function. By differentiation, the probability density function (pdf) of the GONak-G distribution will be obtained as follows:

$$
f(x ; \lambda, \beta, \tau, \eta)=\frac{2 \lambda^{\lambda} \tau g(x) G(x)^{2 \lambda \tau-1}}{\Gamma(\lambda) \beta^{\lambda}\left(1-G(x)^{\tau}\right)^{2 \lambda+1}} e^{-\frac{\lambda}{\beta}\left(\frac{G(x ; \eta)^{\tau}}{1-G(x ; \eta)^{\tau}}\right)^{2}}
$$

The survival function hazard rate function (hrf) is given by

$$
\begin{aligned}
& S(x ; \lambda, \beta, \tau, \eta)=1-\gamma_{*}\left\{\lambda, \frac{\lambda\left[\frac { G ( x ; \eta ) ^ { \tau } } { \beta } \left[\left(1-G(x ; \eta)^{\tau}\right)\right.\right.}{(2}\right\} \\
& h(x ; \lambda, \beta, \tau, \eta)=\frac{\frac{2 \lambda^{\lambda} \tau g(x) G(x)^{2 \lambda \tau-1}}{\Gamma(\lambda) \beta^{\lambda}\left(1-G(x)^{\tau}\right)^{2 \lambda+1}} e^{-\frac{\lambda}{\beta}\left(\frac{G(x ; \eta)^{\tau}}{1-G(x ; \eta)^{\tau}}\right)^{2}}}{1-\gamma_{*}\left\{\lambda, \frac{\lambda\left[\frac { G ( x ; \eta ) ^ { \tau } } { \beta } \left[\left(1-G(x ; \eta)^{\tau}\right)\right.\right.}{(1-G}\right]^{2}}
\end{aligned}
$$

\section{THE GENERALIZED ODD NAKAGAMI- FRECHET (GONak-Fr)}

In this section, we study generalized odd Nakagami Frechet (GONakFr) distributions. Consider the Frechet distribution with cdf and pdf given by: 


$$
G(x ; \rho, v)=e^{-\left(\frac{\rho}{x}\right)^{v}}
$$

and

$$
g(x ; \rho, v)=\frac{\rho^{v} v}{x^{v+1}} e^{-\left(\frac{\rho}{x}\right)^{v}}
$$

Then the pdf of the GONak-Fr distribution is given by

$$
f(x ; \lambda, \beta, \tau, \eta)=\frac{2 \lambda^{\lambda} \tau \rho^{v} v e^{-2 \lambda \tau\left(\frac{\rho}{x}\right)^{v}}}{x^{v+1} \Gamma(\lambda) \beta^{\lambda}\left(1-e^{-\tau\left(\frac{\rho}{x}\right)^{v}}\right)^{2 \lambda+1}} e^{-\frac{\lambda}{\beta}\left(e^{\tau\left(\frac{\rho}{x}\right)^{v}}-1\right)^{-2}}
$$

\subsection{Investigation of the Proposed GONak-Fr Distribution}

To show that the proposed GONak-Fr distribution is a pdf, we proceed as follows:

$$
\int_{0}^{\infty} \frac{2 \lambda^{\lambda} \tau \rho^{v} v e^{-2 \lambda \tau\left(\frac{\rho}{x}\right)^{v}}}{x^{\nu+1} \Gamma(\lambda) \beta^{\lambda}\left(1-e^{-\tau\left(\frac{\rho}{x}\right)^{v}}\right)^{2 \lambda+1}} e^{-\frac{\lambda\left(e^{\tau\left(\frac{\rho}{x}\right)^{v}}-1\right.}{{ }^{2}}} \partial x
$$

Let $u=\frac{\lambda}{\beta}\left(e^{\tau\left(\frac{\rho}{x}\right)^{v}}-1\right)^{-2}$

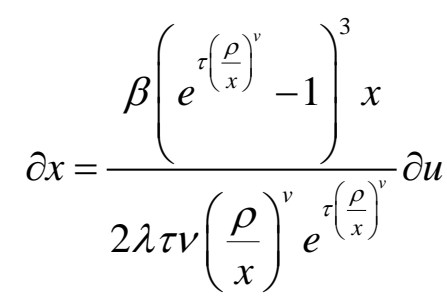

Eq. (9) becomes

$$
\frac{\lambda^{\lambda-1}}{\Gamma(\lambda) \beta^{\lambda-1}} \int_{0}^{\infty}\left(\frac{\beta u}{\lambda}\right)^{\lambda-1} e^{-u} \partial u
$$

then integral in Eq. (10) becomes

$$
\frac{1}{\Gamma(\lambda)} \int_{0}^{\infty} u^{\lambda-1} e^{-u} \partial u=\frac{1}{\Gamma(\lambda)} \Gamma(\lambda)=1
$$

Hence Generalized Odd Nakagami-Frechet Distribution is pdf. 
Various forms of shapes are observed, showing the great flexibility of GONak-Fr distribution.

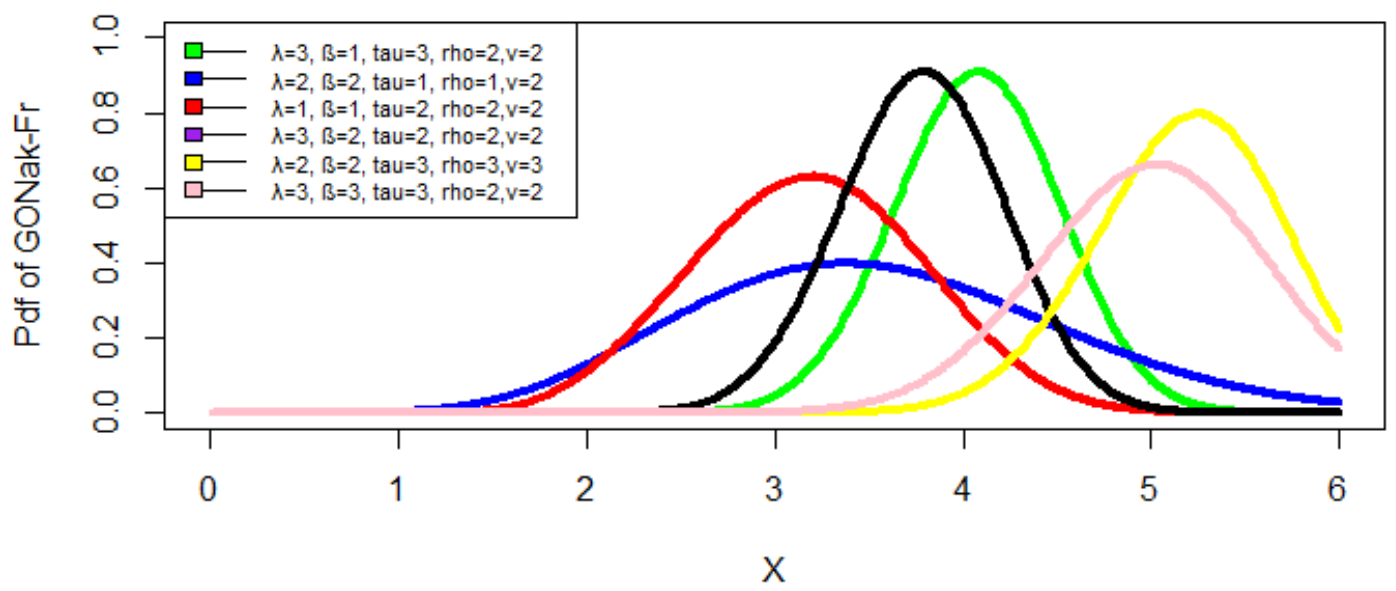

Figure 1. GONak-Fr density function

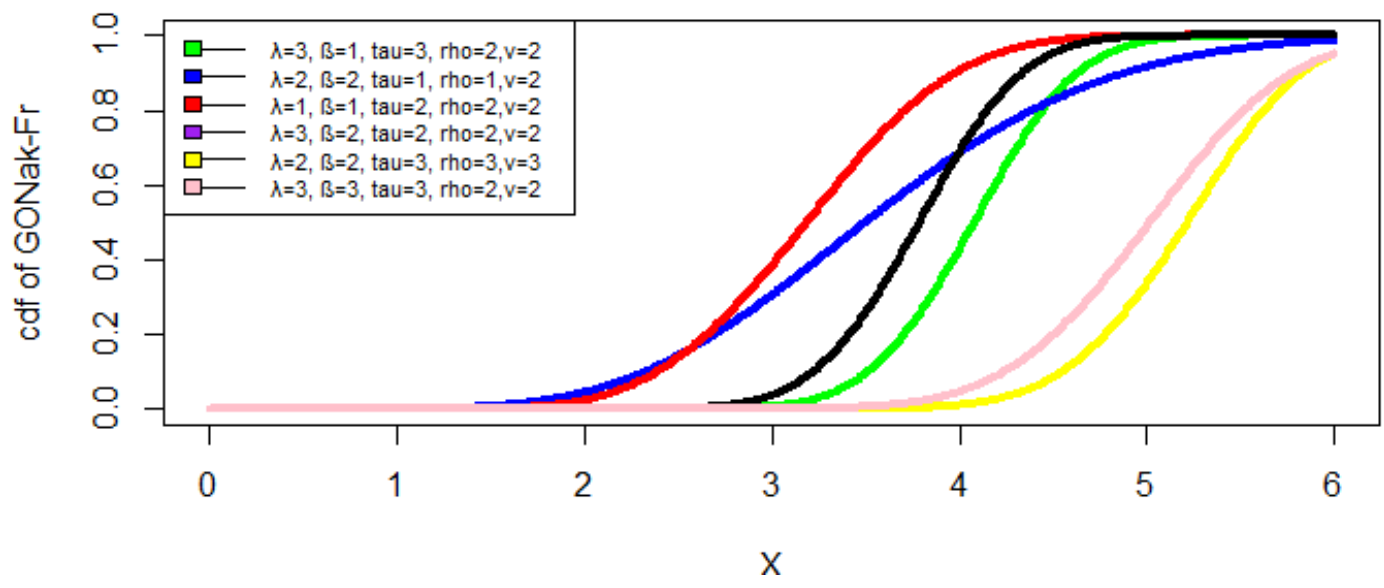

Figure 2. GONak-Fr distribution function

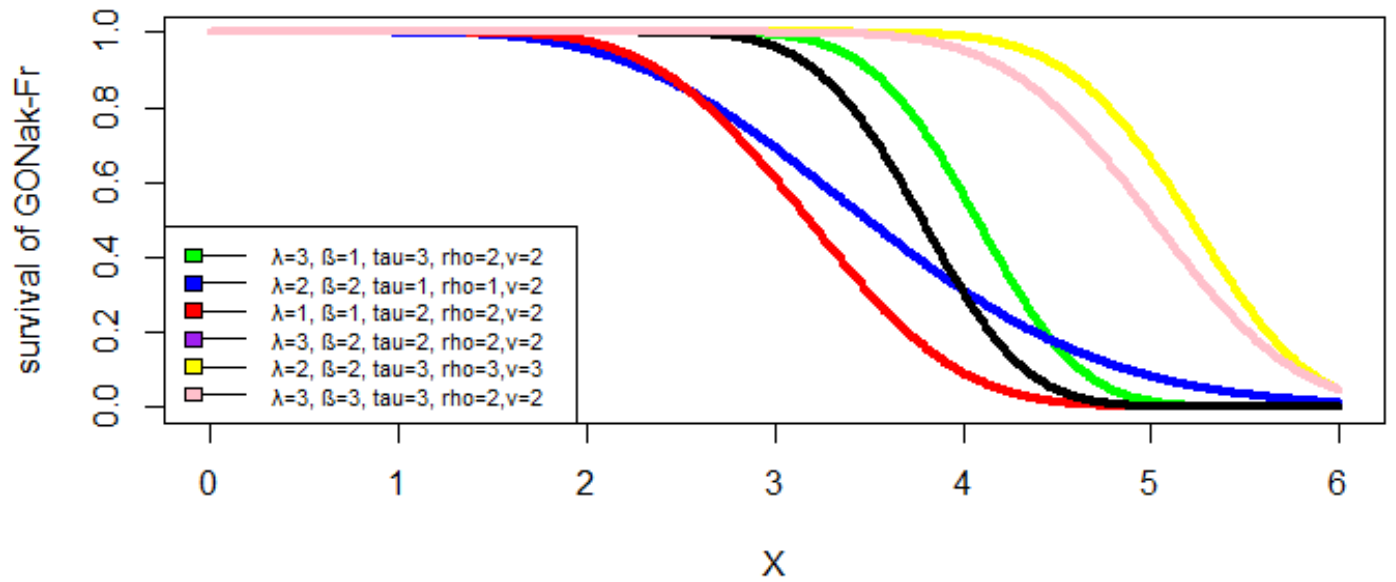

Figure 3. GONak-Fr survival function 


\subsection{Shape of the Crucial Functions}

The shapes of the pdf of the GONak-G family can be defined analytically. The critical points of the GONak-G are given by Eq. (3) are the roots of the resulting equation:

$$
\frac{g(x ; \eta)^{\prime}}{g(x ; \eta)}+(2 \lambda \tau-1) \frac{g(x ; \eta)}{G(x ; \eta)}+(2 \lambda-1) \frac{\tau g(x ; \eta) G(x ; \eta)}{\left(1-G(x ; \eta)^{\tau}\right)}-\frac{2 \lambda \tau g(x ; \eta) G(x ; \eta)^{2 \tau-1}}{\beta\left(1-G(x ; \eta)^{\tau}\right)^{3}}=0
$$

\subsection{Linear Representation}

In this subsection, we provide a very useful linear representation for the GONak-G density function.

We obtain an expansion for density function defined in Eq. (3) as follows: using Taylor expansion the GONak-G pdf becomes

$$
f(x ; \lambda, \beta, \tau, \eta)=\frac{2 \lambda^{\lambda} \tau g(x) G(x)^{2 \lambda \tau-1}}{\Gamma(\lambda) \beta^{\lambda}\left(1-G(x)^{\tau}\right)^{2 \lambda+1}} \sum_{i=0}^{\infty} \frac{(-1)^{i}}{i !}\left(\frac{\lambda}{\beta}\right)^{i}\left[\frac{G(x)^{\tau}}{1-G(x)^{\tau}}\right]^{2 i}
$$

Using generalized binomial series we obtain

$$
\left(1-G(x)^{\tau}\right)^{-[2(\lambda+i)+1]}=\sum_{j=0}^{\infty}\left(\begin{array}{c}
2(\lambda+i)+j \\
j
\end{array}\right) G(x)^{j \tau}
$$

therefore, inserting Eq. (13) in Eq. (12) the GONak-G pd f will take the following form

$$
f(x ; \lambda, \beta, \tau, \eta)=\frac{2 \tau}{\Gamma(\lambda)} \sum_{i, j=0}^{\infty} \frac{(-1)^{i}}{i !}\left(\frac{\lambda}{\beta}\right)^{\lambda+i}\left(\begin{array}{c}
2(\lambda+i)+j \\
j
\end{array}\right) g(x) G(x)^{2 \tau(i+\lambda)+j \tau-1}
$$

this can be written as

$$
\begin{aligned}
& f(x ; \lambda, \beta, \tau, \eta)=\frac{2 \tau}{\Gamma(\lambda)} \sum_{i, j=0}^{\infty} \pi_{i, j} g(x) G(x)^{2 \tau(i+\lambda)+j \tau-1} \\
& \pi_{i, j}=\frac{2 \tau}{\Gamma(\lambda)} \frac{(-1)^{i}}{i !}\left(\frac{\lambda}{\beta}\right)^{\lambda+i}\left(\begin{array}{c}
2(\lambda+i)+j \\
j
\end{array}\right)
\end{aligned}
$$

then, 


$$
f(x ; \lambda, \beta, \tau, \eta)=\sum_{i, j=0}^{\infty} \omega_{i, j} \ell_{2 \tau(i+\lambda)+j \tau}(x)
$$

where

$$
\begin{aligned}
& \omega_{i, j}=\frac{\pi_{i, j}}{2 \tau(i+\lambda)+j \tau}, \\
& \ell_{2 \tau(i+\lambda)+j \tau}(x)=[2 \tau(i+\lambda)+j \tau] g(x ; \eta) G(x ; \eta)^{2 \tau(i+\lambda)+j \tau-1}
\end{aligned}
$$

Eq. (17) is the infinite linear representation of the GONak-G pdf in terms of the exponentiated generated distribution. Similarly, the $c d f$ of the GONak-G family can also be expressed as a linear combination of exponentiated generated $c d f$ given by

$$
F(x ; \lambda, \beta, \tau, \eta)=\sum_{i, j=0}^{\infty} \pi_{i, j} L_{2 \tau(i+\lambda)+j \tau}(x)
$$

where,

$L_{2 \tau(i+\lambda)+j \tau}(x)=G(x ; \eta)^{2 \tau(i+\lambda)+j \tau}$ is the $c d f$ of the exponentiated generated family with the power parameter $2 \tau(i+\lambda)+j \tau$.

\section{MATHEMATICAL AND STATISTICAL PROPERTIES}

In this section, we provide some mathematical properties of the GONak-G distribution.

\subsection{Moments}

The $r^{\text {th }}$ moment of GONak-G family can be obtained from $p d f$ in Eq. (17) as follows

$$
\mu_{r}^{\prime}=\omega_{i, j} \sum_{i, j=0}^{\infty} \int_{0}^{\infty} x^{r} \ell_{2 \tau(i+\lambda)+j \tau}(x) \partial x
$$

that is,

$$
\mu_{r}^{\prime}=\omega_{i, j} I_{r, 2 \tau(i+\lambda)+j \tau,} \quad r=1,2,3, \ldots
$$

where

$$
I_{r, 2 \tau(i+\lambda)+j \tau}=\sum_{i, j=0}^{\infty} \int_{0}^{\infty} x^{r} \ell_{2 \tau(i+\lambda)+j \tau}(x) \partial x
$$


Finally, the mean and variance of the GONak-G family is given by

$$
E(X)=\omega_{i, j} I_{1,2 \tau(i+\lambda)+j \tau}
$$

and

$$
\operatorname{Var}(X)=\omega_{i, j} I_{2,2 \tau(i+\lambda)+j \tau}-\left[\omega_{i, j} I_{1,2 \tau(i+\lambda)+j \tau,}\right]^{2}
$$

\subsection{Moment Generating Function}

The moment generating function of GONak-G family can be obtained from Eq. (20) as follows:

$$
M_{(X)}(t)=\sum_{r=0}^{\infty} \frac{t^{r} \mu_{r}^{\prime}}{r !}=\sum_{r=0}^{\infty} \frac{t^{r} \omega_{i, j} I_{r, 2 \tau(i+\lambda)+j \tau}}{r !}
$$

\subsection{Probability Weighted Moments}

[15] stated that for a random variable $X$, the probability weighted moments ( $p w m$ ) is given by:

$$
\xi_{s, r}=E\left[X^{s} F(x)^{r}\right]=\int_{-\infty}^{\infty} x^{s} F(x)^{r} f(x) \partial x
$$

The PWM of GONak-G is obtained by substituting (2) and (17) into (24)

$$
\xi_{s, r}=\int_{0}^{\infty} x^{s} \gamma_{*}\left\{\lambda, \frac{\lambda}{\beta}\left[\frac{G(x ; \eta)^{\tau}}{\left(1-G(x ; \eta)^{\tau}\right)}\right]^{2}\right\} \sum_{i, j=0}^{\infty} \omega_{i, j} \ell_{2 \tau(i+\lambda)+j \tau}(x) \partial x
$$

using an expansion of incomplete gamma ratio function leads to

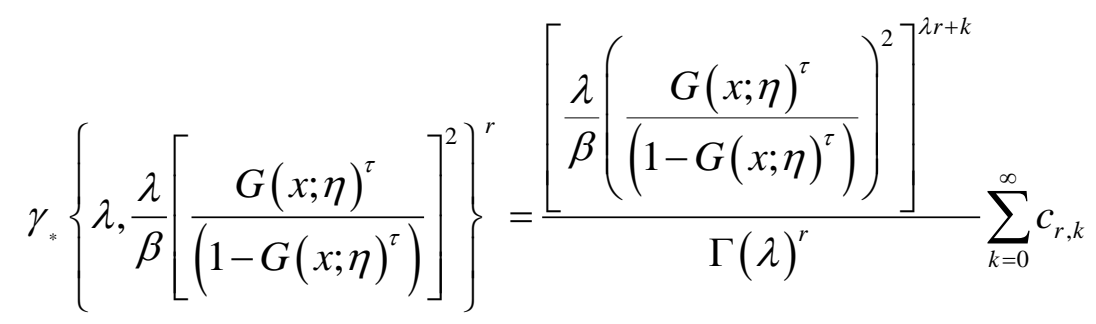

using generalized binomial one can obtain 


$$
\left(1-G(x ; \eta)^{\tau}\right)^{-2(\lambda r+k)}=\sum_{n=0}^{\infty}\left(\begin{array}{c}
2(\lambda r+k)+n-1 \\
n
\end{array}\right) G(x ; \eta)^{n \tau}
$$

substituting Eq. (27) into Eq. (26) leads to

$$
=\frac{\lambda^{\lambda r+k}}{\Gamma(\lambda)^{r} \beta^{\lambda r+k}} \sum_{k, n=0}^{\infty}\left(\begin{array}{c}
2(\lambda r+k)+n-1 \\
n
\end{array}\right) c_{r, k} G(x ; \eta)^{\tau[2(\lambda r+k)+n]}
$$

using generalized binomial one can obtain

$$
G(x ; \eta)^{\tau[2(\lambda r+k)+n]}=\sum_{l=0}^{\infty} \sum_{q=0}^{l}(-1)^{l+q}\left(\begin{array}{c}
\tau[2(\lambda r+k)+n]-1 \\
l
\end{array}\right)\left(\begin{array}{l}
l \\
q
\end{array}\right) G(x ; \eta)^{q}
$$

substituting Eq. (29) into Eq. (28) leads to

$$
\begin{aligned}
= & \frac{\lambda^{\lambda r+k}}{[\Gamma(\lambda)]^{r} \beta^{\lambda r+k}} \sum_{k, n=0}^{\infty} \sum_{l=0}^{\infty} \sum_{q=0}^{l}(-1)^{l+q}\left(\begin{array}{c}
2(\lambda r+k)+n-1 \\
n
\end{array}\right)\left(\begin{array}{c}
\tau[2(\lambda r+k)+n] \\
l
\end{array}\right)\left(\begin{array}{l}
l \\
q
\end{array}\right) \\
& c_{r, k} G(x ; \eta)^{q}
\end{aligned}
$$

replacing $\sum_{l=0}^{\infty} \sum_{q=0}^{l}$ and $\sum_{l=q}^{\infty} \sum_{q=0}^{\infty}$ one can obtain

$$
\begin{aligned}
&= \frac{\lambda^{\lambda r+k}}{[\Gamma(\lambda)]^{r} \beta^{\lambda+k}} \sum_{k, n=0}^{\infty} \sum_{l=q}^{\infty} \sum_{q=0}^{\infty}(-1)^{l+q}\left(\begin{array}{c}
2(\lambda r+k)+n-1 \\
n
\end{array}\right)\left(\begin{array}{c}
\tau[2(\lambda r+k)+n])\left(\begin{array}{l}
l \\
q
\end{array}\right) \\
l
\end{array} \quad c_{r, k} G(x ; \eta)^{q}\right. \\
& F(x)^{r}=\sum_{q=0}^{\infty} \Lambda_{q} G(x ; \eta)^{q}
\end{aligned}
$$

where,

$$
\Lambda_{q}=\frac{\lambda^{\lambda r+k}}{[\Gamma(\lambda)]^{r} \beta^{\lambda r+k}} \sum_{k, n=0}^{\infty} \sum_{l=q}^{\infty}(-1)^{l+q}\left(\begin{array}{c}
2(\lambda r+k)+n-1 \\
n
\end{array}\right)\left(\begin{array}{c}
\tau[2(\lambda r+k)+n] \\
l
\end{array}\right)\left(\begin{array}{l}
l \\
q
\end{array}\right) c_{r, k}
$$

Finally, the $P W M$ of GONak-G is obtained by 


$$
\zeta_{s, r}=\sum_{i, j, q=0}^{\infty} \Lambda_{q} \omega_{i, j} \ell_{s, q+2 \tau(i+\lambda)+j \tau}(x)
$$

\subsection{Entropies}

In this subsection, Renyi entropy will be mentioned as an important measure of uncertainty. The Rényi entropy of a random variable $X$ is defined mathematically as follows:

$$
I_{R}(\delta)=\frac{1}{1-\delta} \log \left(\int_{0}^{\infty} f^{\delta}(x) \partial x\right)
$$

By applying the binomial theory and exponential expansion in the $p d f(3)$, then the $p d f f^{\delta}(x)$ can be expressed as follows

$$
f^{\delta}(x)=\sum_{p, k=0}^{\infty} t_{p, k} g(x)^{\delta} G(x)^{2 \tau(\lambda \delta+p)-\delta+\tau k}
$$

where,

$$
t_{p, k}=\left(\frac{2 \tau}{\Gamma(\lambda)}\right)^{\delta} \frac{(-1)^{p}}{p !} \delta^{p}\left(\frac{\lambda}{\beta}\right)^{\lambda \delta+p}\left(\begin{array}{c}
\delta(2 \lambda+1)+2 p+k-k \\
k
\end{array}\right)
$$

Therefore, the Rényi entropy of GONak-G family is obtained by using (34) as follows

$$
I_{R}(\delta)=\frac{1}{1-\delta} \log \left\{\sum_{p, k=0}^{\infty} t_{p, k} \int_{0}^{\infty} g(x)^{\delta} G(x)^{2 \tau(\lambda \delta+p)-\delta+\tau k} \partial x\right\}
$$

The $\delta$-entropy is defined by

$$
H_{\delta}(X)=\frac{1}{1-\delta} \log \left\{1-\int_{0}^{\infty} f^{\delta}(x) \partial x\right\}
$$

Therefore, the $\delta$-entropy of GONak-G family is obtained by using (37) as follows

$$
H_{\delta}(X)=\frac{1}{1-\delta} \log \left\{1-\sum_{p, k=0}^{\infty} t_{p, k} \int_{0}^{\infty} g(x)^{\delta} G(x)^{2 \tau(\lambda \delta+p)-\delta+\tau k} \partial x\right\}
$$




\subsection{Order Statistics}

Let $x_{1}, x_{2}, \ldots, x_{n}$ be an independent random sample from a distribution function, $F(x)$, with an associated probability density function, $f(x)$. Then, the probability density function of the ith order statistics, $x_{(i)}$, is given by:

$$
f_{X_{(j)}}(x)=\frac{n !}{(j-1) !(n-j) !} \sum_{z=0}^{n-j}(-1)^{z}\left(\begin{array}{c}
n-j \\
z
\end{array}\right) f_{X}(x)\left[F_{X}(x)\right]^{z+j-1}
$$

The pd $\mathrm{f}$ of $r^{\text {th }}$ order statistic from GONak-G distribution is obtained by substituting equation (2) and (3) into (39)

$$
\begin{aligned}
f_{X_{(j)}}(x)= & \frac{n !}{(j-1) !(n-j) !} \sum_{z=0}^{n-j}(-1)^{z}\left(\begin{array}{c}
n-j \\
z
\end{array}\right) \frac{2 \lambda^{\lambda} \tau g(x) G(x)^{2 \lambda \tau-1}}{\Gamma(\lambda) \beta^{\lambda}\left(1-G(x)^{\tau}\right)^{2 \lambda+1}} e^{-\frac{\lambda^{2}}{\beta}\left(\frac{G(x ; \eta)^{\tau}}{1-G(x ; \eta)^{\tau}}\right)^{2}} \\
& \gamma_{*}\left\{\lambda, \frac{\lambda}{\beta}\left[\frac{G(x ; \eta)^{\tau}}{\left(1-G(x ; \eta)^{\tau}\right)}\right]^{2}\right\}^{z+j-1}
\end{aligned}
$$

We now use the series expansion for the incomplete gamma ratio function and substituting into Eq. (40) lead to

$$
\begin{aligned}
f_{X_{(j)}}(x)= & \frac{n !}{(j-1) !(n-j) !}\left(\frac{\lambda}{\beta}\right)^{2 \lambda+v} \sum_{z=0}^{n-j} \sum_{s=0}^{\infty} \sum_{i=s}^{\infty} \sum_{v=0}^{\infty} \frac{(-1)^{z+i+s+v}}{(\lambda+v) v ! \Gamma(\lambda)}\left(\begin{array}{c}
n-j \\
z
\end{array}\right)\left(\begin{array}{c}
z+j-1 \\
i
\end{array}\right)\left(\begin{array}{l}
i \\
s
\end{array}\right) \\
& \frac{2 \tau g(x) G(x)^{4 \lambda \tau+v-1}}{\Gamma(\lambda)\left(1-G(x)^{\tau}\right)^{4 \lambda+v+1}} e^{-\frac{\lambda}{\beta}\left(\frac{G(x ; \eta)^{\tau}}{1-G(x ; \eta)^{\tau}}\right)^{2}}
\end{aligned}
$$

\subsection{Quantile Function}

By considering (2) quantile function (qf) $X$ is obtained as follows:

$$
\begin{aligned}
& u=\gamma_{*}\left\{\lambda, \frac{\lambda}{\beta}\left[\frac{G(x ; \eta)^{\tau}}{\left(1-G(x ; \eta)^{\tau}\right)}\right]^{2}\right\} \\
& u \Gamma(\lambda)=\gamma\left\{\lambda, \frac{\lambda}{\beta}\left[\frac{G(x ; \eta)^{\tau}}{\left(1-G(x ; \eta)^{\tau}\right)}\right]^{2}\right\}
\end{aligned}
$$




$$
G(x ; \eta)=\left\{\frac{\left[\frac{\lambda}{\beta} \gamma^{-1}(\lambda, u \Gamma(\lambda))\right]^{\frac{1}{2}}}{1+\left[\frac{\lambda}{\beta} \gamma^{-1}(\lambda, u \Gamma(\lambda)]^{\frac{1}{2}}\right.}\right\}^{\frac{1}{\tau}}
$$

The quantile function of GONak-Fr distribution is given by:

$$
x=\left\langle\frac{-1}{\rho^{v}} \ln \left\{\frac{\left[\frac{\lambda}{\beta} \gamma^{-1}(\lambda, u \Gamma(\lambda)]^{\frac{1}{2}}\right.}{1+\left[\frac{\lambda}{\beta} \gamma^{-1}(\lambda, u \Gamma(\lambda)]^{\frac{1}{2}}\right.}\right\}^{\frac{1}{\tau}}\right\rangle^{\frac{-1}{v}}
$$

\subsection{Estimation of GONak-G Family}

This subsection deals with the maximum likelihood estimators of the unknown parameters for the GONak-G family of distributions based on complete samples of size n. Let $X_{1}, X_{2}, \ldots, X_{n}$ be observed values from the GONak-G family with a set of parameter $\Theta=(\lambda, \beta, \tau, \eta)^{\prime}$. The log-likelihood function for parameter vector $\Theta=(\lambda, \beta, \tau, \eta)^{\prime}$. is obtained from equation (3) as follows

$$
\begin{gathered}
\ell(\Theta)=n \ln 2+n \lambda \ln \lambda-n \ln \tau-n \ln \Gamma(\lambda)-n \lambda \ln \beta+\sum_{i=0}^{n} \ln g(x ; \eta)+(2 \lambda-1) \\
\sum_{i=0}^{n} \ln G(x ; \eta)-(2 \lambda+1) \sum_{i=0}^{n} \ln \left[1-G(x ; \eta)^{\tau}\right]-\frac{\lambda}{\beta} \sum_{i=0}^{n}[W(x ; \eta)]^{2}
\end{gathered}
$$

where $W(x ; \eta)=\frac{G(x ; \eta)^{\tau}}{1-G(x ; \eta)^{\tau}}$

The components of the score function $U(\Theta)=\left(U_{\lambda}, U_{\beta}, U_{\tau}, U_{\eta}\right)$ are given by 


$$
\begin{aligned}
U_{\lambda}= & n \ln \lambda-n-n \Psi(\lambda)-n \ln \beta+2 \tau \sum_{i=0}^{n} \ln G(x ; \eta)-2 \sum_{i=0}^{n} \ln \left[1-G(x ; \eta)^{\tau}\right] \\
& -\frac{\sum_{i=0}^{n}[W(x ; \eta)]^{2}}{\beta} \\
U_{\beta}= & -\frac{n \lambda}{\beta}+\frac{\lambda \sum_{i=0}^{n}[G(x ; \eta)]^{2}}{\beta^{2}}=0 \\
U_{\tau}= & \frac{u}{\tau}+2 \lambda \sum_{i=0}^{n} \ln G(x ; \eta)+\frac{(2 \lambda+1) \sum_{i=0}^{n} G(x ; \eta)^{\tau} \ln G(x ; \eta)}{\left[1-G(x ; \eta)^{\tau}\right]}-\frac{\lambda}{\beta} \sum_{i=0}^{n} \frac{G(x ; \eta)^{\tau} \ln G(x ; \eta)}{\left[1-G(x ; \eta)^{\tau}\right]^{2}}=0 \\
U_{\eta}= & \sum_{i=0}^{n} \frac{g^{\eta}}{g(x ; \eta)}+(2 \lambda-1) \sum_{i=0}^{n} \frac{G^{\eta}}{G(x ; \eta)}+(2 \lambda+1) \sum_{i=0}^{n} \frac{G^{\eta} G(x ; \eta)^{\tau-1}}{1-G(x ; \eta)^{\tau}} \\
& -\frac{\lambda}{\beta} \sum_{i=0}^{n} \frac{G^{\eta} G(x ; \eta)^{\tau-1} \tau}{\tau}=0 \\
{\left[1-G(x ; \eta)^{\tau}\right]^{2} } &
\end{aligned}
$$

where $g^{\eta}=\frac{\partial g(x ; \eta)}{\partial \eta}$ and $G^{\eta}=\frac{\partial G(x ; \eta)}{\partial \eta}$.

Setting $U_{\lambda}, U_{\beta}, U_{\tau}, U_{\eta}$ equate to zero and solving the equations simultaneously result in the ML estimates $\hat{\Theta}=(\hat{\lambda}, \hat{\beta}, \hat{\tau}, \hat{\eta})$. These estimates cannot be solved algebraically and statistical software can be used to solve them numerically via iterative technique.

\section{RESULTS AND DISCUSSION}

In this section, the fitting of GONakG and some generated families of distribution. We present two applications to real life data set to illustrate the potentiality of the GONak-G family of distribution. To compare its performance, we consider Generalize Odd Nakagami Frechet (GONakFr) to other generated models. The Akaike information criterion (AIC), Consistent Akaikes Information Criterion (CAIC), Bayesian information criterion (BIC), AndersonDarling (A), Kolmogorov Smirnov test (K.S), and the P-Value of K.S test, have been chosen for the comparison of the models.

The first data set which contains 76 observation and is recently used by [16], [17], and [18] it represents the life of fatigue fracture of Kevlar 373/epoxy subjected to constant pressure at 90 percent stress level until all had failed. The data is as follows: 0.0251, 0.0886, 0.0891, 0.2501, $0.3113,0.3451,0.4763,0.5650,0.5671,0.6566,0.6748,0.6751,0.6753,0.7696,0.8375$, 
$0.8391,0.8425,0.8645,0.8851,0.9113,0.9120,0.9836,1.0483,1.0596,1.0773,1.1733$, $1.2570,1.2766,1.2985,1.3211,1.3503,1.3551,1.4595,1.4880,1.5728,1.5733,1.7083$, $1.7263,1.7460,1.7630,1.7746,1.8275,1.8375,1.8503,1.8808,1.8878,1.8881,1.9316$, $1.9558,2.0048,2.0408,2.0903,2.1093,2.1330,2.2100,2.2460,2.2878,2.3203,2.3470$, $2.3513,2.4951,2.5260,2.9911,3.0256,3.2678,3.4045,3.4846,3.7433,3.7455,3.9143$, 4.8073, 5.4005, 5.4435, 5.5295, 6.5541, 9.0960 .

The second set which contains 30 observation and is recently used by [19] and [20] it represents successive values of March precipitation (in inches) in Minneapolis/St Paul. The data are as follows: $0.77,1.74,0.81,1.20,1.95,1.20,0.47,1.43,3.37,2.20,3.00,3.09,1.51,2.10,0.52$, 1.62, 1.31, 0.32, 0.59, 0.81, 2.81, 1.87, 1.18, 1.35, 4.75, 2.48, 0.96, 1.89, 0.90, 2.05.

Tables 1 and 2 display a summary of the MLEs and values of goodness-of-fit measures for the GONak-Fr model and other different models, respectively. As you see, the GONak-Fr is selected as the best model with all the criteria among all the fitted models.

Table 1. MLEs and Goodness-of-fit measures for the first data set

\begin{tabular}{|c|c|c|c|c|c|c|c|c|}
\hline Models & MLE & $-\ell$ & AIC & CAIC & $\mathrm{BIC}$ & A & K.S & P Value \\
\hline GONakFr & $\begin{array}{l}0.8940793 \\
0.2295717 \\
1.8947034 \\
0.5202491 \\
0.3507287\end{array}$ & 119.7429 & 249.4857 & 250.34281 & 261.1394 & 1.739569 & 0.49246 & $2.22 \mathrm{e}-16$ \\
\hline EWW & $\begin{array}{l}1.8638979 \\
1.5739533 \\
0.7065971 \\
0.3125043 \\
0.9306594\end{array}$ & 124.915 & 259.83 & 260.6872 & 271.4837 & 1.092538 & 0.14126 & 0.08724 \\
\hline OGGFr & $\begin{array}{l}1.6021457 \\
1.5297831 \\
0.5340209 \\
0.4477832\end{array}$ & 125.614 & 259.228 & 259.7914 & 268.5509 & 1.183529 & 0.093698 & 0.488 \\
\hline OGGW & $\begin{array}{l}1.2061588 \\
1.3700706 \\
0.5225529 \\
0.6039366\end{array}$ & 124.733 & 257.466 & 258.0294 & 266.7889 & 1.115889 & 0.13597 & 0.1095 \\
\hline
\end{tabular}


Table 2. MLEs and Goodness-of-fit measures for the second data set

\begin{tabular}{|c|c|c|c|c|c|c|c|c|}
\hline Models & MLE & $-\ell$ & AIC & CAIC & BIC & A & K.S & P Value \\
\hline GONakFr & $\begin{array}{l}0.3309079 \\
0.8366780 \\
1.9417631 \\
0.7704069 \\
1.1089862\end{array}$ & 34.56199 & 79.12398 & 81.62398 & 86.12997 & 0.6313438 & 0.22 & 0.1096 \\
\hline EWW & $\begin{array}{l}1.5264454 \\
0.6836198 \\
1.3895067 \\
0.6091976 \\
0.6701214\end{array}$ & 39.20578 & 88.41156 & 90.91156 & 95.41754 & 0.2347084 & 0.10255 & 0.9106 \\
\hline OGGFr & $\begin{array}{l}1.6076918 \\
0.8512776 \\
1.3064885 \\
0.8217882\end{array}$ & 37.89086 & 83.78171 & 85.38171 & 89.3865 & 0.1032816 & 0.060615 & 0.9999 \\
\hline OGGW & $\begin{array}{l}0.5533399 \\
1.7371373 \\
1.3685022 \\
0.9848482\end{array}$ & 37.98979 & 83.97959 & 85.57959 & 89.58438 & 0.1259235 & 0.089259 & 0.9706 \\
\hline
\end{tabular}

life of fatigue fracture of Kevlar 373/epoxy

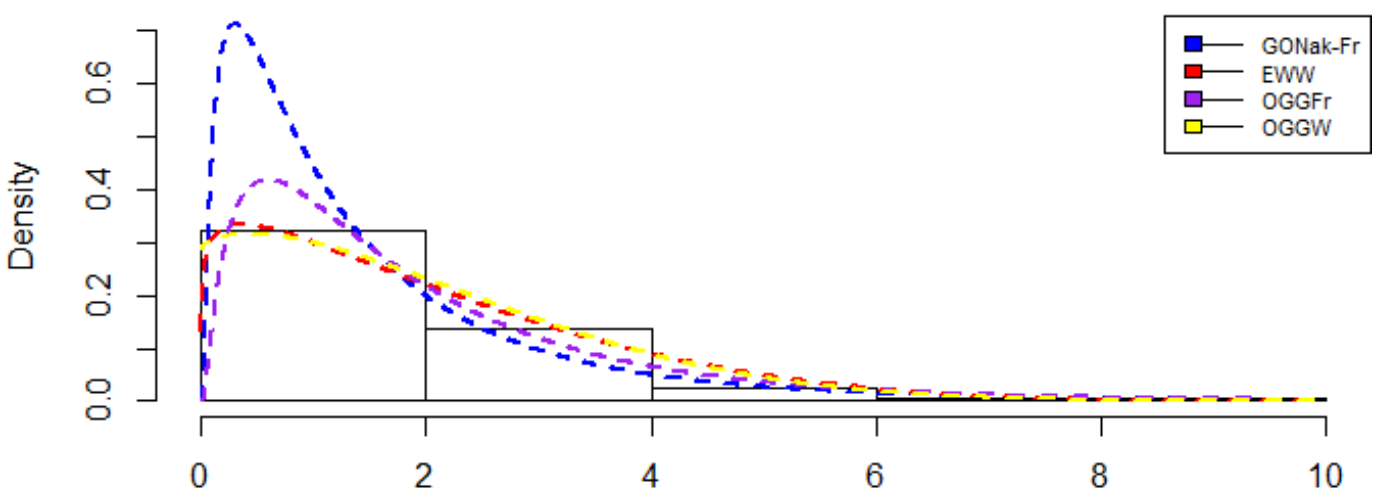

Figure 4. Histogram and estimated pdf for the life of fatigue fracture

\section{CONCLUSIONS}

In this article, we proposed a new Generalized Odd Nakagami (GONak-G) family of distributions, with a special focus on the Frechet distribution as parent distribution. The distribution is named Generalized Odd Nakagami Frechet distribution. The pdf, cdf, survival and hazard function was derived. Additionally, some of the mathematical properties of the new class including quantile, moments, probability weighted moment, order statistics and entropies were also derived. The model parameters were estimated using the maximum likelihood estimation method. Finally, two applications on real life data set are presented to illustrate the potentiality of the proposed model. The new distribution was found to provide a better fit than its competitors. 


\section{REFERENCES}

[1] Eugene, N., Lee, C. and Famoye, F. (2002). Beta-normal distribution and its applications. Communication in Statistics-Theory and Methods, 31: 497-512.

[2] Cordeiro, G.M. and De Castro, M. (2011). A new family of generalized distributions. Journal of Statistical Computation and Simulation, 81: 883-893.

[3] Olanrewaju, I., Shittu, O.I. and Adepoju K.A. (2013). On the beta-nakagami distribution. Progress in Applied Mathematics, 5(1):49-58.

[4] Bourguignon, M., Silva, R.B. and Cordeiro, G.M. (2014). The Weibull-G family of probability distributions. Journal of Data Science, 12(1): 53-68.

[5] Hassan, A.S. and Hemeda, S.E. (2016). The additive Weibull-g family of probability distributions. International Journals of Mathematics and its Applications, 4: 151-164.

[6] Pescim, R. R., Gauss, M., Cordeiro, G.M., Demetrio, C.G.B., Ortega, E.M.M. and Nadarajah, S. (2012). The new class of Kummer beta generalized distributions. SORT-Statistics and Operations Research Transactions, 36(2):153-180.

[7] Cordeiro, G.M., Ortega, M.M.E. and Da Cunha, C.C.D. (2013). The exponentiated generalized class of distributions. Journal of Data Science, 11(1):1-27.

[8] Zografos, K. and Balakrishnan, N. (2009). On families of beta- and generalized gamma-generated distributions and associated inference. Statistical Methodology, 6: 344-362.

[9] Ristic, M.M. and Balakrishnan, N. (2012). The gamma-exponentiated exponential distribution. Journal of Statistical Computation and Simulation, 82:1191-1206.

[10] Alexander, C., Cordeiro, G.M., Ortega, E.M.M. and Sarabia, J.M. (2012). Generalized beta-generated distributions. Computational Statistics \& Data Analysis, 56(6):1880-1897.

[11] Amini, M., Mostafaee, M.S. and Ahmadi, J. (2014). Log-gamma-generated families of distributions. Statistics, 48(4):913-932.

[12] Handique, L. and Chakraborty, S. (2017). A new beta generated Kumaraswamy marshall-olkin-g family of distributions with applications. MJS, 36(3):157-174.

[13] Alizadeh, M., Cordeiro, G.M., De Brito, E. and Demetrio, C.G.B. (2015). The beta marshall-olkin family of distributions. Journal of Statistical Distributions and Applications, 2(1):4

[14] Hamzeh Torabi, H. and Montazeri, N.H. (2014). The logistic-uniform distribution and its applications. Communications in Statistics-Simulation and Computation, 43(10):2551-2569.

[15] Oguntunde, P. E., Adejumo, A. O. and Owoloko, E. A. (2017). On the exponentiated generalized inverse exponential distribution. Proceedings of the World Congress on Engineering, Vol. I

[16] Barlow, R.E., Toland, R.H. and Freeman, T. (1984). A Bayesian analysis of stress-rupture life of kevlar 49/epoxy spherical pressure vessels. In Proc. Conference on Applications of Statistics Ao, Marcel Dekker, New York.

[17] Gomez, M. Y., Bolfarine, H., and Gomez, W.H. (2014). A new extension of the exponential distribution. Revista Colombiana de Estadistica, 37(1):25-34.

[18] Greenwood, J.A., Landwehr, J.M. and Matalas, N.C. (1979). Probability weighted moments: definitions and relations of parameters of several distributions expressible in inverse form. Water Resources Research, 15: 10491054. 
[19] Hinkley, D. (1977). On quick choice of power transformations. Journal of the Royal Statistical Society, Series (c), Applied Statistics, 26:67-69.

[20] Elgrahy, M. and Hassan, A. (2019). Exponentiated weibull weibull distribution. Statistical properties and applications. Gazi University Journal of Science, 32(2):616-635. 